\begin{tabular}{|c|c|}
\hline Title & $\begin{array}{l}\text { A coustic stop bands of surface and bulk modes in two-dimensional phononic lattices consisting of aluminum and a } \\
\text { polymer }\end{array}$ \\
\hline Author(s) & Tanaka, Y ukihiro; Tamura, Shin-ichiro \\
\hline Citation & $\begin{array}{l}\text { PHY SICALL REV IEW B, 60(19), } 1329413297 \\
\text { https:/doi.org/10.1103/Phy sRevB.60.13294 }\end{array}$ \\
\hline Issue Date & $1999-11$ \\
\hline Doc URL & http:/hdl .handle.net/2115/5854 \\
\hline Rights & Copyright @ 1999 A merican Physical Society \\
\hline Type & article \\
\hline File Information & PRB60-19.pdf \\
\hline
\end{tabular}

Instructions for use 


\title{
Acoustic stop bands of surface and bulk modes in two-dimensional phononic lattices consisting of aluminum and a polymer
}

\author{
Yukihiro Tanaka and Shin-ichiro Tamura \\ Department of Applied Physics, Hokkaido University, Sapporo 060-8628, Japan
}

(Received 1 June 1999)

\begin{abstract}
Spatial and frequency distributions of forbidden bands of both surface and bulk acoustic waves are studied theoretically for two-dimensional (2D) periodic elastic structures consisting of aluminum and polymer. The surface is perpendicular to the $2 \mathrm{D}$ periodic arrays of circular cylinders embedded in a background material. The dispersion relations of the surface and bulk modes with wave vectors parallel to the surface are calculated for triangular lattices, and the stop band distributions are plotted in a form relevant to the comparison with ultrasound imaging experiments. [S0163-1829(99)03043-X]
\end{abstract}

There has been growing interest in recent years in the study of photonic crystals, i.e., periodic, dielectric structures. ${ }^{1,2}$ The existence of complete band gaps (photonic band gaps) of electromagnetic waves in these structures extending throughout the Brillouin zone can lead to a variety of phenomena of both fundamental and practical interest. The analogy between photons and phonons suggests the consideration of periodic elastic composites of two or more vibrating materials called phononic crystals. So far, several authors have developed for such composites theories of acoustic band structure for bulk vibrations, ${ }^{3-5}$ and the predicted acoustic stop bands have been observed experimentally. ${ }^{6}$

A recent subject concerning phononic crystals is the acoustic mode localized near the truncated surface of the structure. ${ }^{7}$ Such localized surface waves in both one- and two-dimensional periodic elastic structures have recently been studied theoretically by the present authors for the combinations of materials (GaAs and AlAs) with small acoustic mismatch. ${ }^{8-10}$ The surface wave branch is predicted to exist below the lowest bulk wave branch, in general, though it can also appear inside the bulk band for specific directions of propagation. Moreover, the folding effect characteristic of a periodic system is found for the surface wave branches near the Brillouin-zone boundary.

Recently, ultrasound imaging experiments with twodimensional (2D) phononic crystals have been performed by Vines and Wolfe. ${ }^{11}$ Using line-focus transducers, they generated and detected ultrasonic waves with a well-defined wave vector, and the occurrence of band gaps (stop bands) in the transmission spectrum along the surface has been observed. ${ }^{12,13}$ The samples employed constitute of polycrystalline aluminum and polymer (Crystalbond), which are elastically isotropic. The structures composed of these materials are characterized by a large acoustic mismatch. Vines and Wolfe applied a simple model for interpreting the stop band images obtained. More explicitly, they analyzed the surface acoustic waves in terms of a single bulk mode decoupled from other two modes with an appropriate scaling of the velocity. ${ }^{11}$ Actually, the surface acoustic waves in the system studied consist of three modes of vibrations.

In this paper we present more detailed calculations of the dispersion relations of acoustic waves (both surface and bulk modes) propagating along the surfaces of $2 \mathrm{D}$ periodic struc- tures consisting of aluminum and Crystalbond. We also study the stop band distributions of both the surface and bulk acoustic waves in these $2 \mathrm{D}$ phononic crystals.

In the system composed of a 2D periodic array of different materials, the mass density $\rho$ and elastic stiffness tensor $c_{i j m n}$ are position dependent. The equations of motion for the lattice displacement $\mathbf{u}(\mathbf{r}, t)$ are thus given by

$$
\rho\left(\mathbf{x}_{\|}\right) \ddot{u}_{i}=\partial_{j}\left[c_{i j m n}\left(\mathbf{x}_{\|}\right) \partial_{n} u_{m}\right] \quad(i=1,2,3),
$$

where $\mathbf{r}=\left(\mathbf{x}_{\|}, z\right)=(x, y, z)$, and we take the $z$ axis perpendicular to the free surface (parallel to the $x-y$ plane). The system is assumed to be periodic in the $x-y$ plane [see the inset of Fig. 1(a)]. The summation convention over repeated indices is assumed in Eq. (1). The bulk wave frequencies are obtained by solving eigenvalue equations derived from Eq. (1), but for surface wave solutions, we further need the boundary conditions at the surface $z=0$,

$$
\left.\left.T_{i 3}\right|_{z=0} \equiv c_{i 3 m n}\left(\mathbf{x}_{\|}\right) \partial_{n} u_{m}\right|_{z=0}=0 \quad(i=1,2,3),
$$

where the surface is assumed to be stress free. The eigenfrequency and the complex wave number $k_{z}$ (governing the localization of the wave near the surface) of the surface acoustic mode are obtained by simultaneously solving Eqs. (1) and (2).

For solving these equations, we employ the plane-wave expansion, ${ }^{3,4}$ which was also applied to the study of the phonon focusing in 1D periodic superlattices. ${ }^{9,14}$ In this scheme the lattice displacement and the position dependent mass density and elastic constants are

$$
\begin{gathered}
\mathbf{u}(\mathbf{r}, t)=\sum_{\mathbf{G}} e^{i\left(\mathbf{k}_{\|}+\mathbf{G}\right) \cdot \mathbf{x}_{\|}-i \omega t} \mathbf{a}_{\mathbf{G}}(z), \\
\alpha\left(\mathbf{x}_{\|}\right)=\sum_{\mathbf{G}} e^{i \mathbf{G} \cdot \mathbf{x}_{\|}} \alpha_{\mathbf{G}},
\end{gathered}
$$

where $\alpha=\left(\rho, c_{i j m n}\right), \mathbf{k}_{\|}=\left(k_{x}, k_{y}\right)$ is a Bloch wave vector, and $\mathbf{G}$ is a $2 \mathrm{D}$ reciprocal-lattice vector. Note that $\rho$ and $c_{i j m n}$ do not depend on $z$ because of the homogeneity of the system along this direction. In solving Eqs. (1) and (2), we put $\mathbf{a}_{\mathbf{G}}(z)=\mathbf{A}_{\mathbf{G}} e^{i k_{z} z}$. For a surface wave solution, all of the wave numbers $k_{z}$ 's should be complex. 

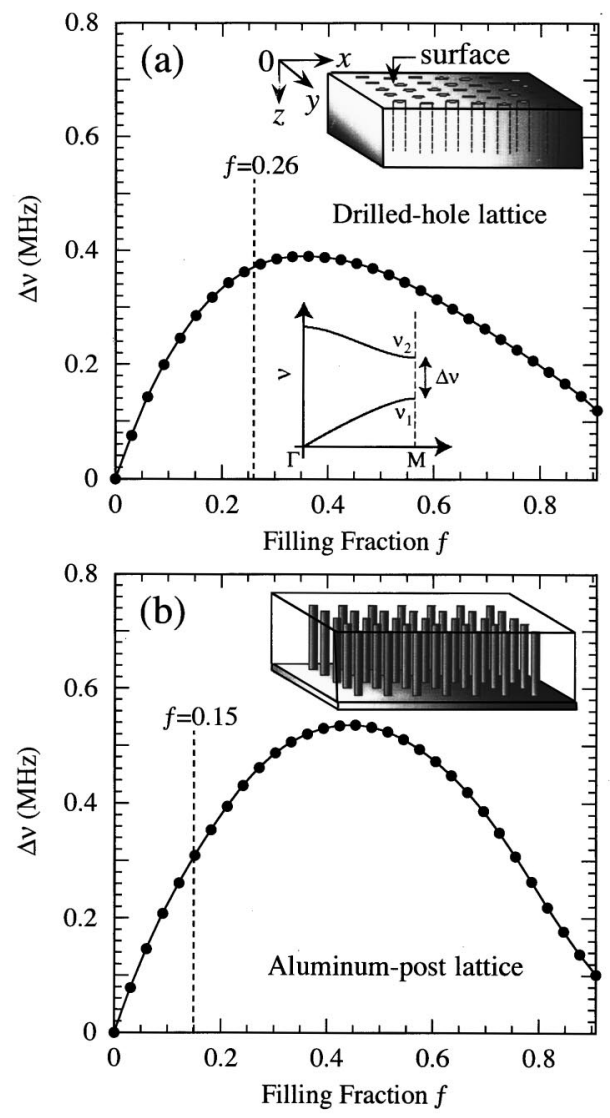

FIG. 1. Magnitude of the frequency gap $\left(\Delta \nu=\nu_{2}-\nu_{1}\right)$ of the lowest single mode (shear vertical wave) at the $M$ point of the Brillouin-zone boundary versus filling fraction $f$ of two-dimensional (2D) triangular lattices consisting of $\mathrm{Al}$ and Crystalbond ( $\xi$ $=\mathrm{v}_{s} / \mathrm{v}_{T}=0.93$ for Crystalbond). (a) Drilled-hole lattice and (b) aluminum-post lattice. The vertical dashed lines indicate the filling fractions chosen for the ultrasound imaging experiment by Vines and Wolfe (Ref. 11). Schematics of 2D phononic lattices are also shown in the insets.

Now we consider the system where elastic circular cylinders (denoted by $A$ ) of radius $r_{0}$ are embedded periodically in a background material (denoted by $B$ ) forming a triangular lattice with lattice spacing $a$. In this structure the reciprocal-lattice vector is $\mathbf{G}=\left[2 \pi N_{1} / a, 2 \pi\left(2 N_{2}\right.\right.$ $\left.\left.-N_{1}\right) / \sqrt{3} a\right]$ with $N_{1}$ and $N_{2}$ integers, and the Fourier coefficients are given by $\alpha_{\mathbf{G}}=f \alpha_{A}+(1-f) \alpha_{B}$ for $\mathbf{G}=0$ and $\alpha_{\mathbf{G}}=\left(\alpha_{A}-\alpha_{B}\right) F(\mathbf{G})$ for $\mathbf{G} \neq 0$, where $f=2 \pi r_{0}^{2} / \sqrt{3} a^{2}$ is the filling fraction that defines the cross-sectional area of a cylinder relative to a unit-cell area, and $F(\mathbf{G})$ $=2 f J_{1}\left(|\mathbf{G}| r_{0}\right) /|\mathbf{G}| r_{0}$ with $J_{1}(x)$ a Bessel function.

According to Vines and Wolfe, ${ }^{11}$ we consider the drilledhole and aluminum-post lattices (shown schematically in Fig. 1) where the cylinders consist of Crystalbond and aluminum for the former and latter lattices, respectively. The sound velocities of polycrystalline aluminum are found in literature, but those of Crystalbond (CB) are not known. For Crystalbond we only know the surface wave velocity $\left(\mathrm{V}_{s}\right)$ measured by Vines and Wolfe, i.e., $v_{s}=2.26 \times 10^{5} \mathrm{~cm} / \mathrm{s} .{ }^{11}$ In an isotropic substrate the ratio $\xi=\mathrm{v}_{s} / \mathrm{v}_{T}$ of the velocity of the Rayleigh surface wave to that of the transverse bulk wave $\left(\mathrm{v}_{T}\right)$ takes $\xi=0.874-0.955$ depending on the ratio of the transverse sound velocity to that of the longitudinal wave. In
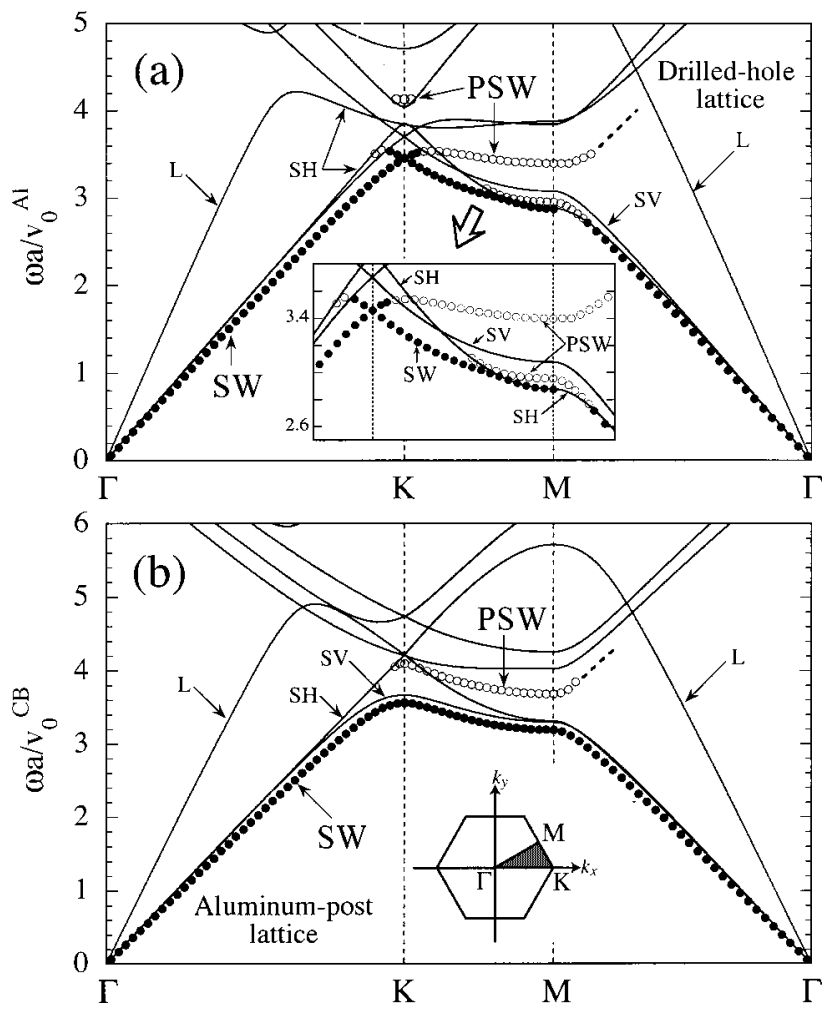

FIG. 2. Dispersion relations of surface acoustic waves (SW, dots), bulk longitudinal (L), shear vertical (SV, polarized parallel to the cylinder axis), and shear horizontal ( $\mathrm{SH}$, polarized normal to the cylinder axis) acoustic waves (solid lines) propagating along the surface of two-dimensional triangular lattices consisting of $\mathrm{Al}$ and Crystalbond. ( $a$ is the lattice spacing, $\mathrm{v}_{0}^{\mathrm{Al}}=3.09 \times 10^{5} \mathrm{~cm} / \mathrm{s}$ and $\mathrm{v}_{0}^{\mathrm{CB}}=2.43 \times 10^{5} \mathrm{~cm} / \mathrm{s}$ are the transverse sound velocities in $\mathrm{Al}$ and Crystalbond, and $\xi=\mathrm{v}_{s} / \mathrm{v}_{T}=0.93$ is assumed for Crystalbond.) (a) Drilled-hole lattice with filling fraction $f=0.26$ and (b) aluminumpost lattice with $f=0.15$. Also plotted by open circles are the pseudosurface waves (PSW's). All these branches correspond to the waves with wave vector perpendicular to the cylinder axis. The irreducible part of the Brillouin zone and the magnification of the central region of (a) are also illustrated.

the present study, we have assumed three representative values $\xi=0.90,0.93$, and 0.95 for Crystalbond, but the results are insensitive to $\xi$ for these three chosen values. ${ }^{15}$

In Figs. 1(a) and 1(b) the magnitudes of the lowest frequency gap $\Delta \nu$ of the bulk transverse branch at the $M$ point of the Brillouin zone are plotted for the drilled-hole and aluminum-post lattices as a function of the filling fraction $f$. We see that the largest frequency gap is obtained at $f$ $\sim 0.35$ for the drilled-hole lattice and at $f \sim 0.5$ for the aluminum-post lattice. The filling fraction $f=0.26$ chosen by Vines and Wolfe ${ }^{11}$ for the drilled-hole sample is thus close to the value for which the largest frequency gap is obtained, but at $f=0.15$ chosen for the aluminum-post sample, a rather small gap is realized.

(a) Dispersion relations in drilled-hole lattices. In this structure the low-density polymer is filled in the circular cylinders $(A)$ in a high-density aluminum $(B)$ background. The assumed filling fraction is $f=0.26$, i.e., the value used in the experiment. Figure 2(a) shows the low-frequency part of the dispersion curves of both the bulk (with wave vectors parallel to the $x-y$ plane) and surface waves along the boundary of 
the irreducible part of the Brillouin zone shown in the inset of Fig. 2(b). Along the $\Gamma-K$ direction, the folded surface wave branch is found near the $K$ point though no frequency gap is recognized. This degeneracy of the branches at the $K$ point is also seen for the bulk branches and stems from the fact that the waves at three equivalent $K$ points of the Brillouin-zone boundary of the hexagonal shape are coupled to each other, and the symmetry of the lattice yields the same values for the low-lying two frequencies. [The third frequency is found inside bulk band as a pseudosurface wave (PSW) explained below.]

Along the zone boundary $K-M$ line, the frequency gap is open between the normal and folded surface wave branches up to about $5^{\circ}$ rotated from the $K$ point. At larger angles the folded surface wave branch continues to exist as a PSW branch inside bulk band. The PSW is a resonance with bulk waves and is sometimes observed as a surface wave. ${ }^{16}$ For a real $k_{z}$, the boundary condition determinant derived from Eq. (2) only exhibits a dip at the frequency corresponding to the PSW. However, by adding a small and negative imaginary part to $k_{z}$, which represents a small energy radiation into the bulk of the system, this dip goes down to zero.

Interestingly, along the $\Gamma-M$ direction, the surface wave branch intersects the lowest bulk transverse branch near the $M$ point and becomes the PSW branch close to the zone boundary. This branch continues to exist along the $K-M$ line of the zone boundary up to $\sim 17^{\circ}$ rotated from the $K$ point, where the normal surface wave is almost degenerate to the lowest bulk wave.

(b) Dispersion relations in aluminum-post lattices. For this structure the filling fraction $f=0.15$ (the same value as in the experimental sample) is assumed for the calculation. The surface wave branch is found along the whole region of the boundary of the irreducible part of the Brillouin zone slightly below the lowest bulk transverse branch. Contrary to the case of the drilled-hole lattice, the folded surface wave branch is found neither along the $\Gamma-K$ direction nor the $\Gamma-M$ direction. However, PSW branches appear along these directions. Along the $K-M$ line of the zone boundary, a PSW branch is also found inside bulk band, and their frequencies are almost parallel to the ones of the surface wave along the same line. Although no folded surface wave is found in this lattice, there exist PSW branches that act as folded branches of the surface waves. Thus the stop bands between the surface and PSW's exist in this 2D structure.

(c) Stop band distributions. The calculated stop band distributions are shown in Figs. 3(a) and 3(b) for the drilledhole and aluminum-post lattices, respectively. The lowest distribution shows the prohibited frequency regions between the surface wave (SW) and PSW branches (also between the SW and SW, and the PSW and PSW branches for certain directions) along the zone boundary. We see that these SWPSW stop bands extend over all propagation directions in both lattices except at the angle $0^{\circ}$ (and equivalent directions) for the aluminum-post sample. This is not necessarily the case for the bulk waves. Also gaps due to the bulk transverse mode polarized perpendicular to the surface (shear vertical mode) are partly overlapped with the SW-PSW gaps. The incomplete stop bands due to the mixed longitudinal and transverse (shear horizontal mode) modes ${ }^{10}$ exist slightly above these low-lying gaps. All those stop bands are related
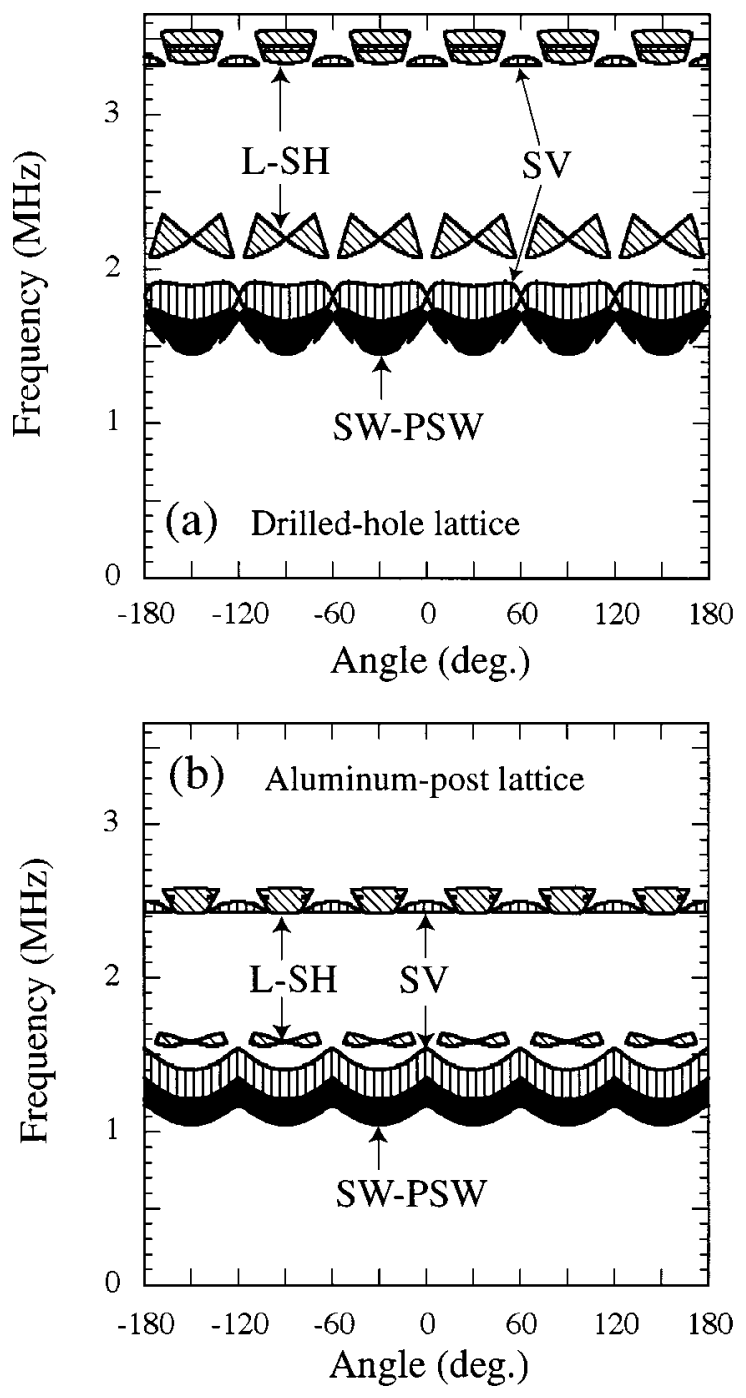

FIG. 3. Distributions of the acoustic stop bands of the surface and pseudosurface waves (SW-PSW, smeared region) and bulk waves [shear vertical, coupled shear horizontal, and longitudinal (L) waves, shaded regions]. (a) Drilled-hole lattice with filling fraction $f=0.26$ and (b) aluminum-post lattice with $f=0.15$. The frequency range shown is the same as that given in the ultrasound images by Vines and Wolfe (Ref. 11). The horizontal axis is the angle in the wave-vector space measured from the $\Gamma-K$ direction.

to the waves with wave vectors parallel to the surface and have sizable magnitudes. The calculated distributions of SWPSW stop bands are well correlated with the ultrasound image for the drilled-hole lattice, but unfortunately, the good coincidence is not obtained for the aluminum-post lattice. We do not know the reason for this discrepancy, but it may be related to the waves reflected from the bottom face of the sample.

To conclude, we have calculated the dispersion relations and stop band distributions of the surface and bulk acoustic waves in 2D phononic crystals consisting of aluminum and a polymer. For the present systems, the folding effect and associated frequency gap in the surface wave dispersion relation are seen only near the $K$ point for the drilled-hole lattice. This is quite different from the results for the surface waves in 2D phononic crystals consisting of GaAs and AlAs with small acoustic mismatch, where the folded surface wave 
branches are found for a wide range of the zone boundary. However, slightly above the surface wave branch, the PSW branches exist inside the bulk band, which seem to act as folded surface wave branches. Thus, the stop bands due to the surface and (folded) pseudosurface waves can be still defined in the phononic crystals studied in the present paper.

In the imaging experiment by Vines and Wolfe, ${ }^{11}$ immersion transducers are used to generate and detect ultrasound signals, that is, the sample surface is covered with liquid. Thus the loading of liquid on the sample surface of phononic crystals should give some effect on the observed images. ${ }^{13,17,18}$ We also note that the elastic constants of some polymers are frequency dependent in the $\mathrm{MHz}$ range, and at the same time the ultrasonic absorption is large. We plan to extend our theory to the liquid loaded phonon crystals with frequency-dependent, complex elastic constants.

The authors are grateful to R. E. Vines and J. P. Wolfe for sending the preliminary data of their imaging experiments. This work was supported in part by a Grant-in-Aid for Scientific Research from the Ministry of Education, Science and Culture of Japan (Grant No. 09640385).
${ }^{1}$ Photonic Band Gaps and Localization, edited by C. M. Soukoulis (Plenum, New York, 1993).

${ }^{2}$ J. D. Joannopoulos, R. D. Meade, and J. N. Winn, Photonic Crystals (Princeton University Press, Princeton, NJ, 1995).

${ }^{3}$ M. S. Kushwaha, P. Halevi, L. Dobrzynski, and B. D. -Rouhani, Phys. Rev. Lett. 71, 2022 (1993).

${ }^{4}$ M. S. Kushwaha, P. Halevi, G. Martines, L. Dobrzynski, and B. D.-Rouhani, Phys. Rev. B 49, 2313 (1994).

${ }^{5}$ M. Sigalas and E. N. Economou, Solid State Commun. 86, 141 (1993).

${ }^{6}$ F. R. Montero de Espinosa, E. Jimenez, and M. Torres, Phys. Rev. Lett. 80, 1208 (1998).

${ }^{7}$ M. Torres, F. R. Montero de Espinosa, D. Garcia-Pablos, and N. Garcia, Phys. Rev. Lett. 82, 3054 (1999).

${ }^{8}$ T. Aono and S. Tamura, Phys. Rev. B 58, 4838 (1998).

${ }^{9}$ T. Aono, Y. Tanaka, and S. Tamura, Physica B 263-264, 98 (1999).

${ }^{10}$ Y. Tanaka and S. Tamura, Phys. Rev. B 58, 7958 (1998); Physica B 263-264, 77 (1999).
${ }^{11}$ R. E. Vines and J. P. Wolfe (unpublished).

${ }^{12}$ R. E. Vines, M. R. Hauser, and J. P. Wolfe, Z. Phys. B 98, 255 (1995).

${ }^{13}$ R. E. Vines, S. Tamura, and J. P. Wolfe, Phys. Rev. Lett. 74, 2729 (1995); 75, 1873(E) (1995).

${ }^{14}$ Y. Tanaka, M. Narita, and S. Tamura, J. Phys.: Condens. Matter 58, 7958 (1998).

${ }^{15}$ The sound velocities used are $\mathrm{v}_{L}=6.37 \times 10^{5} \mathrm{~cm} / \mathrm{s}$ (longitudinal mode), $\mathrm{v}_{T}=3.09 \times 10^{5} \mathrm{~cm} / \mathrm{s}$ for aluminum, and $\mathrm{v}_{S}=2.26$ $\times 10^{5} \mathrm{~cm} / \mathrm{s}$ for the Rayleigh wave velocity in Crystalbond. The mass densities are $\rho_{C B}=1.35 \mathrm{~g} / \mathrm{cm}^{3}$ for Crystalbond and $\rho_{\mathrm{Al}}$ $=2.7 \mathrm{~g} / \mathrm{cm}^{3}$ for aluminum.

${ }^{16}$ G. W. Farnell, in Physical Acoustics VI, edited by W. P. Mason and R. N. Thurston (Academic, New York, 1970), p. 109.

${ }^{17}$ I. A. Viktorov, E. K. Grishchenko, and T. M. Kaekina, Akust. Zh. 9, 162 (1963) [Sov. Phys. Acoust. 9, 131 (1963)].

${ }^{18}$ S. Tamura, R. E. Vines, and J. P. Wolfe, Phys. Rev. B 54, 5151 (1996). 